\title{
Contribution to Modeling a Robust Controller for a Flexible Aircraft
}

\author{
Ranarison Solofo Herizo, Randriamitantsoa Paul Auguste, \\ Randriamitantsoa Andry Auguste, \\ Reziky Zafimarina S.H.Z.T
}

\begin{abstract}
This study uses the Hamilton method to derive the equation of motion of a flexible aircraft. The purpose is to design a robust controller using the $H_{\infty}$ and the loo-shaping methods. The $\boldsymbol{\mu}$ method is used to analyze the system and the $v$ - gap, to compare the controllers.
\end{abstract}

Index Terms--Flexible aircraft, $\mathrm{H}_{\infty}$ algorithm, loop-shaping, $\boldsymbol{\mu}$-analysis, $\boldsymbol{v}$-gap.

\section{INTRODUCTION}

Modeling the motion of a flexible aircraft is more complex than the case of a rigid aircraft. The flexibility of the structure increases the number of the parameters of the equations of motion. The components of the state vector are intensified, and it is complicating the design of an efficient controller for the system. In this article, only the longitudinal flight of a flexible airplane will be developed.

1. EQUATIONS OF MOTION

The function of Lagrange is defined by:

$$
\mathbf{L}=\mathbf{T}-\mathbf{U}
$$

And the Hamiltonian function is defined by:

$$
\mathbf{H}=\mathbf{p}^{\mathbf{T}} \dot{\mathbf{q}}-\mathbf{L}
$$

Where $\mathbf{q}$ represents the generalized coordinates, $\mathbf{T}$ is the kinetic energy, $\mathbf{U}$ is the potential energy and $\mathbf{p}$ is given by:

$$
\mathbf{p}=\frac{\partial \mathbf{L}}{\partial \dot{\mathbf{q}}}
$$

The equations of the motion of the system is given by the Hamilton canonic equations:

$$
\left\{\begin{array}{l}
\dot{\mathbf{q}}=\frac{\partial \mathbf{H}}{\partial \mathbf{p}} \\
\dot{\mathbf{p}}=-\frac{\partial \mathbf{H}}{\partial \mathbf{q}}+\mathbf{Q}
\end{array}\right.
$$

Where $\mathbf{Q}$ is the generalized force applied on the system.

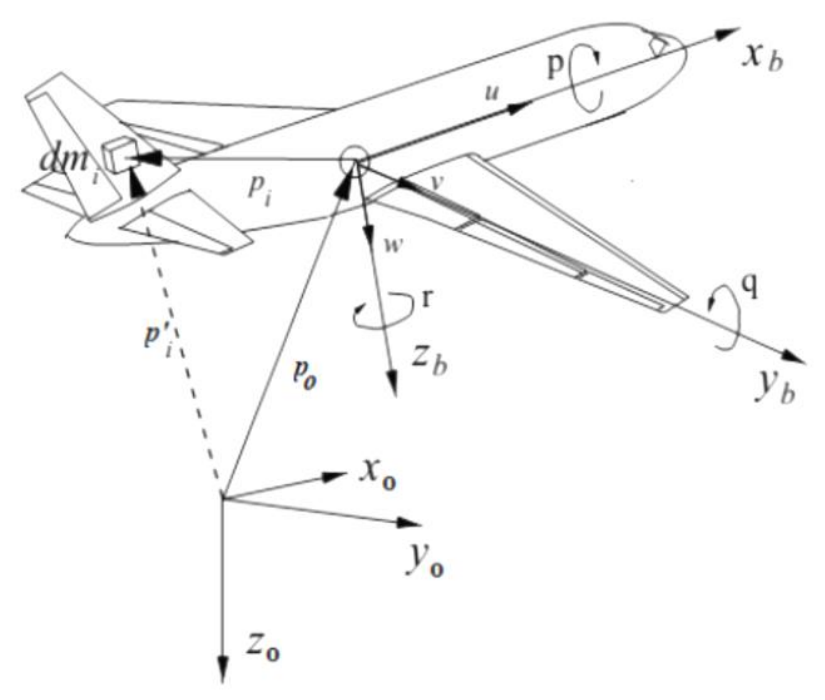

Fig. 1. Flexible aircraft model.

For the flexible aircraft showed in the Fig 1, the equations of energy are:

$$
\begin{gathered}
\mathbf{T}=\frac{1}{2} m\left(u^{2}+v^{2}+w^{2}\right) \\
+\frac{1}{2}\left[\begin{array}{lll}
p & q & r
\end{array}\right][I]\left[\begin{array}{l}
p \\
q \\
r
\end{array}\right]+\frac{1}{2} \sum_{i=1}^{n} M_{i} \dot{\eta}_{i}^{2} \\
\mathbf{U}_{e}=\frac{1}{2} \sum_{i=1}^{n} \omega_{i}{ }^{2} \eta^{2}{ }_{i} M_{i}
\end{gathered}
$$

$$
\mathbf{U}_{g}=\left[\begin{array}{lll}
m g \sin \theta & -\sin \phi \cos \theta-\cos \phi \cos \theta
\end{array}\right]\left[\begin{array}{l}
x \\
y \\
z
\end{array}\right]
$$

$\left[\begin{array}{lll}u & v & w\end{array}\right]^{T}:$ Velocities vector;

$\left[\begin{array}{lll}p & q & r\end{array}\right]^{T}$ : Angular velocities vector;

$m$ : Vehicle mass;

$g$ : Gravity acceleration;

$\eta_{i}$ : The generalized displacement coordinates of the ith vibration mode;

$\omega_{i}$ : The undamped natural frequency of the ith vibration mode; 
$M_{i}$ : The respective generalized mass of the ith vibration mode;

$I_{x x}, I_{y y}, I_{z z}:$ The moments of inertia;

$I_{x y}, I_{y z}:$ The $x y$ and $y z$ inertial product;

$\phi, \theta$ : The roll and the pitch angle;

Ue: Elastic potential energy;

Ug: Elastic potential energy.

Then the function of Lagrange:

$$
\begin{aligned}
& \mathbf{L}=\frac{1}{2} m\left(u^{2}+v^{2}+w^{2}\right)+\frac{1}{2}\left[\begin{array}{lll}
p & q & r
\end{array}\right][I]\left[\begin{array}{l}
p \\
q \\
r
\end{array}\right]+\frac{1}{2} \sum_{i=l}^{n} M_{i} \dot{\eta}_{i}{ }^{2}- \\
& \frac{1}{2} \sum_{i=1}^{n} \omega_{i}{ }^{2} \eta^{2}{ }_{i} M_{i}-\left[m g \sin \theta-\sin \phi \cos \theta-\cos \phi \cos \theta\left[\begin{array}{l}
x \\
y \\
z
\end{array}\right]\right.
\end{aligned}
$$

And the Hamiltonian function:

$$
\begin{aligned}
& \mathbf{H}=m u \dot{x}+m v \dot{y}+m w \dot{z}-\frac{1}{2} m\left(u^{2}+v^{2}+w^{2}\right)+ \\
& \frac{1}{2}\left[\begin{array}{lll}
p & q & r
\end{array}\right][I]\left[\begin{array}{l}
p \\
q \\
r
\end{array}\right]+\frac{1}{2} \sum_{i=1}^{n} M_{i} \dot{\eta}_{i}^{2} \\
& +\frac{1}{2} \sum_{i=1}^{n} \omega_{i}{ }^{2} \eta^{2}{ }_{i} M_{i}+\left[\begin{array}{lll}
m g \sin \theta & -\sin \phi \cos \theta-\cos \phi \cos \theta
\end{array}\right]\left[\begin{array}{l}
x \\
y \\
z
\end{array}\right]
\end{aligned}
$$

With the Hamilton canonic equations, the longitudinal equation of motionis given by:

$$
\left\{\begin{array}{l}
m[\dot{u}-r v+q w+g \sin \theta]=X \\
m[\dot{w}-q u+p v-g \cos \phi \cos \theta]=Z \\
\dot{q} I_{y y}-\left(\dot{p} I_{x y}+\dot{r} I_{y z}\right)+\left(I_{x x}-I_{z z}\right) p r \\
+\left(p I_{y z}-r I_{x y}\right) q+\left(p^{2}-r^{2}\right) I_{x z}=M \\
\ddot{\eta}_{i}+\omega_{i}{ }^{2} \eta_{i}=\frac{Q_{i}}{M_{i}}
\end{array}\right.
$$

Where: $X, Y, Z$ The longitudinal force, the normal force and the pitching moment;

\section{SYSTEM DESCRIPTIONS AND MODELING}

With some assumption in the parameters and for one vibration mode, the resulting of state-space model for linear control synthesis is given by:

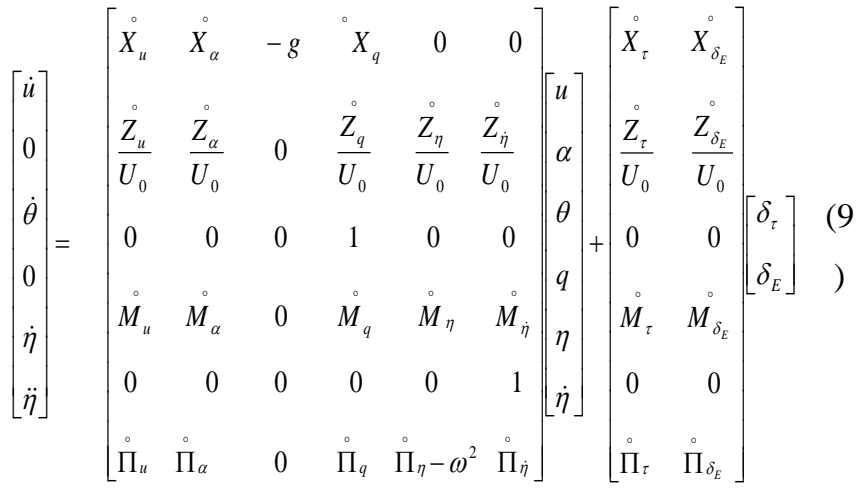

$x^{T}=\left[\begin{array}{lllllll}u & \alpha & \theta & \dot{\theta}=q & \eta & \dot{\eta}\end{array}\right]:$ The state vector;

$\left[\begin{array}{ll}\delta_{\tau} & \delta_{E}\end{array}\right]^{T}:$ Thrust and elevator commands;

$U_{O}$ : Equilibrium longitudinal velocity;

$\stackrel{\circ}{\Pi}$ : Short notation for the derivative of $\frac{\mathbf{Q}_{\mathbf{i}}}{M_{i}}$;

$\stackrel{\circ}{X}, \stackrel{\circ}{Z}, \stackrel{\circ}{M}$ : The derivative notation of $X, Z$ and $M$

3.1. $H_{\infty}$ synthesis

\section{SYNTHESIS}

The problem when designing a controller is to find a controller $\mathrm{K}$ for a system $\mathrm{P}$ (Fig 2) that generates a signal u considering the information from $y$ to mitigate the effect of $w$ on $z$. In fact, the controller is synthesized while minimizing the closed loop norm $\mathrm{w}$ to $\mathrm{z}$.

$$
\left\|F_{l}(P, K)\right\|_{\infty}<\gamma
$$

$\left\|F_{l}(P, K)\right\|_{\infty}$ : The lower linear fractional transformation of $\mathrm{P}$ and $\mathrm{K}$, transfer function from $\mathrm{w}$ to $\mathrm{z}$;

$\gamma:$ A fixed real number.

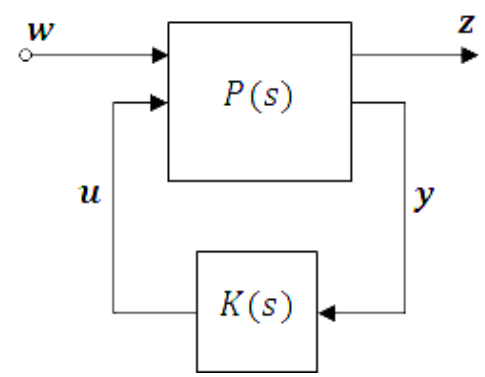

Fig. 2. Standard interconnection for the $\boldsymbol{H}_{\infty}$ synthesis. 


\subsection{Loop shaping synthesis}

Loop shaping procedure shaped the nominal plant using a precompensator $\mathrm{W}_{1}$ and/or a post-compensator $\mathrm{W}_{2}$. The controller is synthesized with minimizing the norm of the McFarlan and Glover stability margin, such that:

$$
\left\|\left[\begin{array}{c}
I \\
K_{\infty}
\end{array}\right]\left(I+P_{s} K_{\infty}\right)^{-1} \tilde{M}_{s}^{-1}\right\|_{\infty} \leq \varepsilon^{-1}
$$

Where $P_{s}=W_{2} P W_{1}$ and $\varepsilon$ an optimal stability margin;

$P_{s}=\tilde{M}_{s}^{-1} \tilde{N}_{l}$ is the coprime factor of $P_{s}$.

The final feedback controller is:

$$
K=W_{1} K_{\infty} W_{2}
$$

\section{4. $\mu$-ANALYSIS}

$\mathrm{M}$ is defined as a transfer function form, $\mathrm{w}$ to $\mathrm{z}$ :

$$
M=F_{1}(P, K)
$$

The structured singular value of a matrix $M$ is defined as:

$$
\mu_{\Delta}=\frac{1}{\min [\bar{\sigma}(\Delta): \operatorname{det}(I-\Delta M)=0]}
$$

$\bar{\sigma}$ denoted the upper singular value and $\Delta$ a set of uncertainty.

The closed-loop system achieves the nominal performance if only if:

$$
\sup _{\Delta}\left(M_{22}\right)<1
$$

The closed-loop system achieves the robust stability if, only if:

$$
\sup _{\Delta}\left(M_{11}\right)<1
$$

The closed-loop system achieves the robust performance if, only if:

$$
\sup \mu_{\Delta}(M)<1
$$

\section{5. v-GAP}

The $v$-gap metric is defined by the quantification of the distance between any two processes in terms of similarity of behavior when connected to a closed loop.

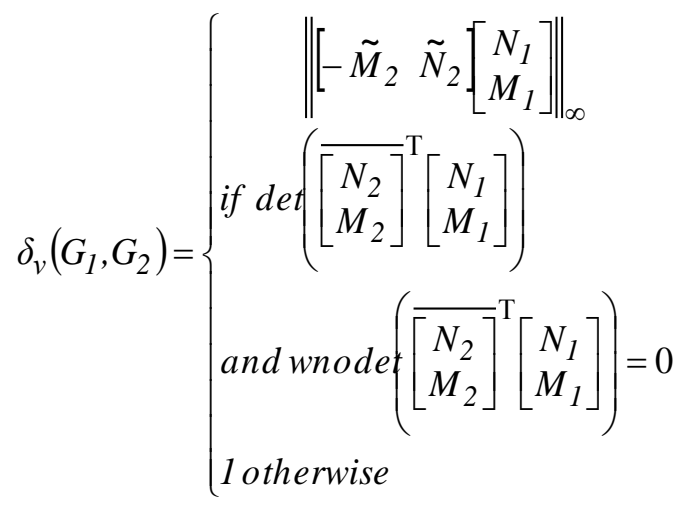

$G_{i}=N_{i} M_{i}^{-1}=\tilde{M}_{i}^{-1} \tilde{N}_{i}:$ The normalized right (left) coprime factorizations of the plants no denoted the winding number.

\section{Simulation}

The large high-speed is adopted as the simulation object. The singular value plot of the system is shown in Fig 3. The peak value is $80 \mathrm{~dB}$ at $0.356 \mathrm{rad} / \mathrm{s}$, it is the necessary to design a controller to stabilize the system.

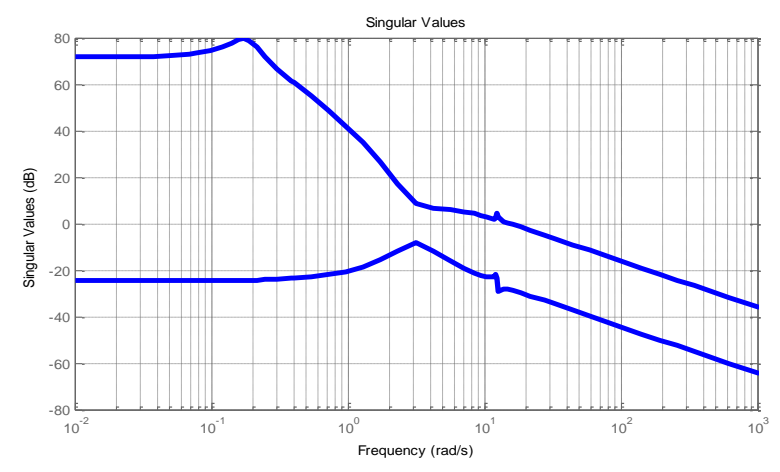

Fig. 3. Diagram of the singular value

Controllers are synthesis with the open-loop bock shown in fig 4. The system is perturbed by an additive uncertainty. The objectives of the design are to maintain stability and performance in presence of a bounded uncertainty.

The weights are selected to maximize disturbance rejection, and minimize wind gust effect and a sensor noise.

$$
W_{1}=1,5 \frac{s+2}{3 s+10}, W_{2}=\frac{12,5}{s+10}
$$




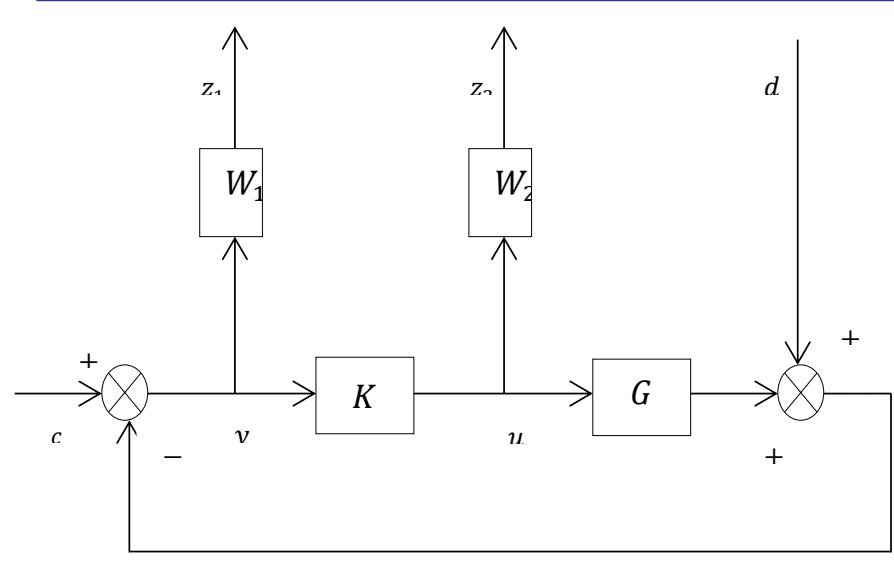

Fig. 4. Close-loop system with additive uncertainty.

The two controllers are obtained:

- $\boldsymbol{H}_{\infty}$ controller:

$$
K_{H}=\left[\begin{array}{ll}
\frac{h_{11}}{d_{h}} & \frac{h_{12}}{d_{h}} \\
\frac{h_{21}}{d_{h}} & \frac{h_{22}}{d_{h}} \\
\frac{h_{31}}{d_{h}} & \frac{h_{32}}{d_{h}} \\
\frac{h_{41}}{d_{h}} & \frac{h_{42}}{d_{h}}
\end{array}\right]
$$

With:

$d_{h}=s^{3}+1,41 s^{2}+0,4838 s+0,04285$

$h_{11}=1,947 \cdot 10^{7} s^{3}-2,89 \cdot 10^{5} s^{2}+9,614 \cdot 10^{7} s+1,91 \cdot 10^{7}$

$h_{12}=-2,345.10^{6} s^{3}+0,0003394 s^{2}-6,505.10^{6} s-1,925.10^{6}$

$h_{21}=-1,029 \cdot 10^{10} s^{3}+5,663 \cdot 10^{9} s^{2}-7,473 \cdot 10^{10} s-7,442 \cdot 10^{11}$

$h_{22}=4,165.10^{10} s^{3}-6,775.10^{8} s^{2}+7,231.10^{9} s+7,78 \cdot 10^{10}$

$h_{31}=4,492.10^{9} s^{3}+1,598.10^{7} s^{2}+4,066.10^{8} s+1,987.1 \theta^{9}$

$h_{32}=1,33.10^{8} s^{3}-1,773.10^{6} s^{2}-4,542.10^{7} s-2,234.10^{8}$

$h_{41}=8,759.10^{10} s^{3}-1,615 \cdot 10^{8} s^{2}+4,456.10^{9} s+3,662.10^{10}$

$h_{42}=-4,752.10^{9} s^{3}+1,983.10^{7} s^{2}-4,543.10^{8} s-3,889.10^{9}$

- Loop shaping controller:

$$
K_{L}=\left[\begin{array}{ll}
\frac{l_{11}}{d_{l}} & \frac{l_{12}}{d_{l}} \\
\frac{l_{21}}{d_{l}} & \frac{l_{22}}{d_{l}} \\
\frac{l_{31}}{d_{l}} & \frac{l_{32}}{d_{l}} \\
\frac{l_{41}}{d_{l}} & \frac{l_{42}}{d_{l}}
\end{array}\right]
$$

With:

$d_{l}=s^{3}+1178 s^{2}+3075 s+2968$

$l_{11}=2,346 \cdot 10^{6} s^{3}+0,02839 s^{2}+0,562 s-0,00102$

$l_{12}=-2,541.10^{5} s^{3}-0,3548 s^{2}-6,977 s-0,2226$

$l_{21}=-1,099.10^{9} s^{3}-8,814.10^{6} s^{2}-0,000182 s-2,992 \cdot 10^{5}$

$l_{22}=1,253 \cdot 10^{8} s^{3}+0,000110 s^{2}+0,002254 s+0,000433 t$

$l_{31}=-3,9 \cdot 10^{9} s^{3}-0,0004008^{2}-0,007566 s-0,002176$

$l_{32}=-3,934.10^{8} s^{3}+0,004978 s^{2}+0,09351 s+0,02911$

$l_{41}=-2,964.10^{9} s^{3}-6,189.10^{5} s^{2}-0,00121 s-0,001066$

$l_{42}=2,307 \cdot 10^{8} s^{3}+0,0007675^{2}+0,01483 s+0,01324$

The $v$-gap of the two controller is:

$$
\delta_{v}\left(K_{H}, K_{L}\right)=0,0024
$$

It mean that the two controller are close.

The close-loop matrix for the $\mu$-analysis (Fig 5) is :

$$
M=\left[\begin{array}{cc}
-W_{2} K S & W_{2} K S \\
-W_{1} S & W_{1} S
\end{array}\right]
$$

Where $S=[1+G K]^{-1}$.

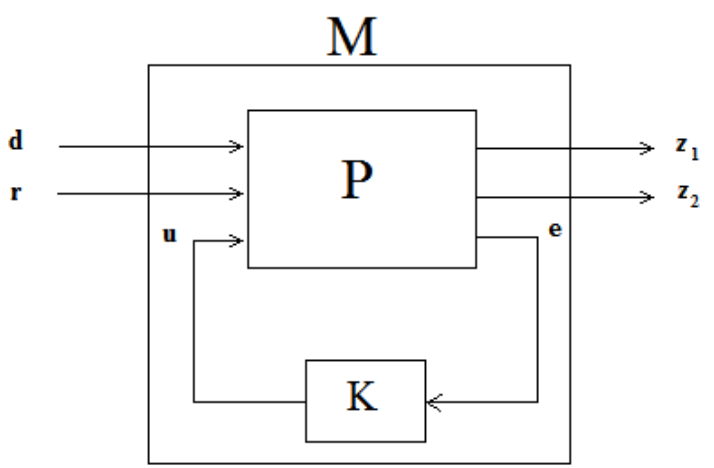

Fig. 5. Close-loop matrix for $\mu$-analysis.

The frequency range of the analysis is $\left[10^{-4} ; 10^{4}\right] \mathrm{rad} / \mathrm{s}$.

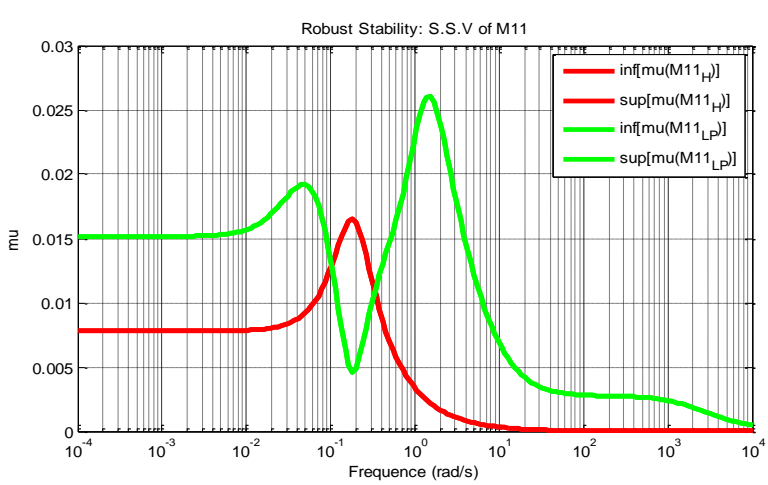

Fig. 6. $\mu$-plot for robust stabilityanalysis 
TABLE 1 RESULTS OF ROBUST STABILITY ANALYSIS

\begin{tabular}{|c|c|c|c|}
\hline $\begin{array}{c}\text { Close- } \\
\text { loop }\end{array}$ & $\begin{array}{c}\omega_{m}(\mathrm{rad} \\
/ \mathrm{sec})\end{array}$ & $\max \left[\mu\left(M_{11}\right)\right]$ & Guaranteed of stability \\
\hline$K_{H}$ & 0,1804 & 0,0165 & $\|\Delta\|_{\infty}<\frac{1}{0,0165}$ \\
\hline$K_{L P}$ & 1.5167 & 0.0260 & $\|\Delta\|_{\infty}<\frac{1}{0.0260}$ \\
\hline
\end{tabular}

The peak value of $\mu\left(M_{11}\right)$ is less than one for each case of closeloop (Table 1, Fig 6). This implies that for all perturbations, $\|\Delta\|_{\infty}<\frac{1}{\mu\left(M_{11}\right)}$ the stability is guaranteed. The guaranteed stability is large for the $H_{\infty}$ algorithm.

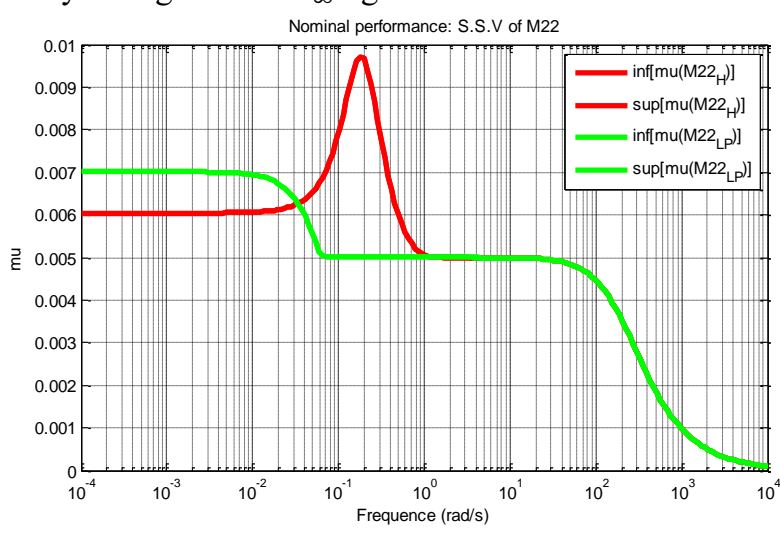

Fig. 7. $\mu$-plot for nominal performance analysis

TABLE 2 RESULTS OF NOMINAL PERFORMANCE ANALYSIS

\begin{tabular}{|c|c|c|}
\hline Close-loop & $\omega_{m}(\mathrm{rad} / \mathrm{sec})$ & $\max \left[\mu\left(M_{22}\right)\right]$ \\
\hline$K_{H}$ & 0,1804 & 0,0097 \\
\hline$K_{L P}$ & 0,0001 & 0,0070 \\
\hline
\end{tabular}

The peak value of $\mu\left(M_{22}\right)$ is less than one for each case of closeloop (Table 2, Fig 7). This implies that for all perturbations, nominal performance was achieved. However the performance specification is better for the close-loop with loop shaping algorithm.

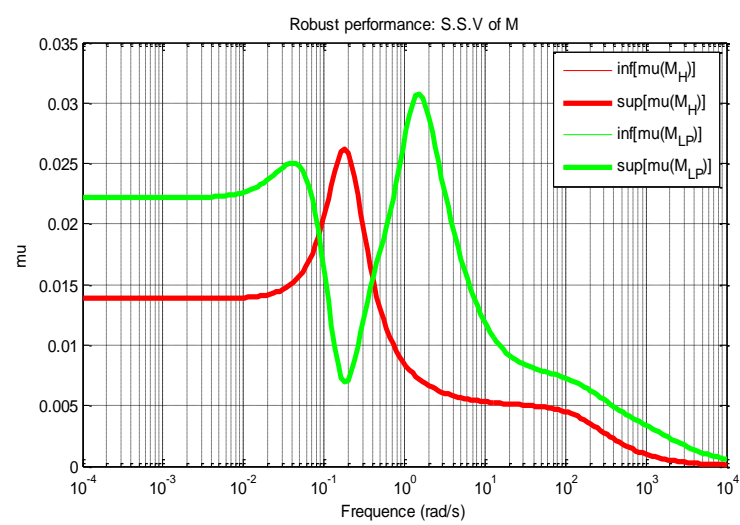

Figure $6 \mu$-plot for robust stability analysis
TABLE 2 RESULTS OF ROBUST STABILITY ANALYSIS

\begin{tabular}{|c|c|c|c|}
\hline $\begin{array}{l}\text { Close- } \\
\text { loop }\end{array}$ & $\begin{array}{l}\omega_{m}(\mathrm{rad} \\
/ \mathrm{sec})\end{array}$ & $\max [\mu(M)]$ & $\begin{array}{c}\text { Guaranteed of } \\
\text { stability }\end{array}$ \\
\hline$K_{H}$ & 0,1804 & 0,0262 & $\|\Delta\|_{\infty}<\frac{1}{0,0262}$ \\
\hline$K_{L P}$ & 1,5167 & 0,0307 & $\|\Delta\|_{\infty}<\frac{1}{0,0307}$ \\
\hline
\end{tabular}

The peak value of $\mu(M)$ is less than one for each case of closeloop (Table 3, Fig 8). This implies that for all perturbations, $\|\Delta\|_{\infty}<\frac{1}{\mu(M)}$ the performance is guaranteed. The guaranteed performance is large for the $H_{\infty}$ algorithm.

\section{CONCLUSION}

The large high speed is instable. However, the two controllers designs, $H_{\infty}$ and loop shaping guarantee a robust stability, and a nominal and robust performance. The two controllers are close in reference of the $v$-gap.

\section{REFERENCES}

[1] L. Meirovitch, "Methods of Analytical Dynamics", Hill, 1970.W.-K. Chen, Linear Networks and Systems. Belmont, Calif.: Wadsworth, pp. 123-135, 1993. (Book style)

[2] M.R. Waszak, D.K. Schmidt, "Flight Dynamics of Aeroelastic Vehicles", Journal of Aircraft, 1988

[3] D.K. Schmidt, "Modern Flight Dynamics", Hill, 2012.

[4] C. Zhu, "Robustness analysis for power systems based on the structured singular value tools and the nu gap metric", PHD, Iowa State University, 2001.

[5] G. Vinnicombe, "Uncertainity and Feedback $H_{\infty}$ loop-shaping and the $v-$ gap metric", Imperial College Press, 2001.

[6] K. Zhou, J.C. Doyle, K. Glover, "Robust and Optimal Control", Prentice Hall, New Jersey, 1996.

[7] K. Zhou, J.Doyle,"Essentials of Robust Control", Prentice Hall, 1999.

RANARISON S. H., received his Master Diploma in Automatic from 2015 at Ecole Supérieure Polytechnique d'Antananarivo (ESPA), University of Antananarivo. Currently he is a $\mathrm{PhD}$ student at University of Antananarivo in the STII,

RANDRIAMITANTSOA P.A., full Professor, Ecole Supérieure Polytechnique d'Antananarivo (ESPA), University of Antananarivo.

RANDRIAMITANTSOA A.A., Doctor, Ecole Supérieure Polytechnique d'Antananarivo (ESPA), University of Antananarivo.

REZIKY Z.S.H.Z.T, Doctor, Ecole Supérieure Polytechnique d'Antananarivo (ESPA), University of Antananarivo. 